\title{
Association between MPO-463G > A polymorphism and cancer risk: evidence from 60 case-control studies
}

\author{
Wen-jun Yang ${ }^{1 \dagger}$, Ming-yue Wang ${ }^{2 \dagger}$, Fu-ze $\mathrm{Pan}^{3+}{ }^{+}$Chen $\mathrm{Shi}^{4}$ and Hong Cen ${ }^{2 *}$
}

\begin{abstract}
Background: Though a number of studies have been conducted to explore the association between myeloperoxidase (MPO)-463G > A polymorphism and cancer risk, the results remain inconsistent. Therefore, we performed a meta-analysis to derive a more systematic estimation of this relationship.

Method: Relevant studies were searched by PubMed, EMBASE, CNKI, Google Scholar, Ovid, and Cochrane library prior to December 2015. The strength of the association between MPO-463G > A polymorphism and cancer risk was estimated by odds ratios (OR) with 95\% confidence interval (95\%Cl). Cumulative analysis was used to evaluate the stability of results through time.

Results: The current analysis consisted of 16,858 cases and 21,756 controls from 60 studies. Pooled results showed that MPO-463G > A polymorphism were associated with the overall decreased cancer susceptibility in all the genetic models included in this study (additive model: $\mathrm{OR}=0.84,95 \% \mathrm{Cl}=0.76-0.94$; allele genetic model: $\mathrm{OR}=0.90,95 \% \mathrm{Cl}=0.840-0$. 954; recessive genetic model: $\mathrm{OR}=0.89,95 \% \mathrm{Cl}=0.83-0.95)$. However, in the stratified analysis of cancer type, the significant results were only found in lung cancer (dominant model: $\mathrm{OR}=0.93,95 \% \mathrm{Cl}=0.87-0.99$ ) and digestive system cancer groups (dominant model: $\mathrm{OR}=0.670 .53-0.84$; allele frequency model $=0.71,95 \% \mathrm{Cl}=0.57-0.87$ ), but not in the blood system cancer or breast cancer group. When we further stratified the digestive system cancer group into digestive tract and digestive gland cancer groups, results showed a significant association between allele $\mathrm{A}$ of MPO-463G > A and digestive gland cancer in all the genetic models (allele frequency model: $\mathrm{OR}=0.63,95 \% \mathrm{Cl}=0.40-0$. 99; additive model: $\mathrm{OR}=0.41,95 \% \mathrm{Cl}=0.23-0.73$; recessive model: $\mathrm{OR}=0.51,95 \% \mathrm{Cl}=0.29-0.89$; dominant model: $\mathrm{OR}=0.58,95 \% \mathrm{Cl}=0.35-0.96)$, digestive tract cancers in allele frequency model $(\mathrm{OR}=0.75,95 \% \mathrm{Cl}=0.59-0.95)$, and dominant model $(\mathrm{OR}=0.72,95 \% \mathrm{Cl}=0.56-0.92)$. When stratified by ethnicity, results demonstrated that the genotype A might be a protect factor for both Caucasians and Asians. In group analysis according to source of controls, significant results were found in population from hospital in all the genetic models. In cumulative analysis, result of allele contrast showed a declining trend and increasingly narrower 95\% overall, while the inclination toward non-significant association with lung cancer risk.
\end{abstract}

Conclusions: This meta-analysis suggested that MPO-463G > A polymorphism was associated with the overall reduced cancer susceptibility significantly. It might be a more reliable predictor of digestive system cancer instead of lung cancer, blood system cancer, and breast cancer. In cumulative analysis, the stable trend indicated that evidence was sufficient to show the association between MPO-463G > A polymorphism and cancer risk.

Keywords: Gene polymorphism, Myeloperoxidase, Meta-analysis, Cancer

\footnotetext{
* Correspondence: hongcen2016@163.com

${ }^{\dagger}$ Equal contributors

${ }^{2}$ Department of Chemotherapy, Affiliated Tumor Hospital of Guangxi Medical

University, Nanning 530021, Guangxi, China

Full list of author information is available at the end of the article
} 


\section{Background}

Cancer is a multifactorial disease resulted from complex interactions between genetic and environmental and its worldwide burden is increasing [1]. Reactive oxidative stress (ROS) is showed to play a significant role in immunological defense, intracellular signaling, and intercellular communication [2]. An increased ROS is considered to be mutagenic and carcinogenic, and it may result in cell damage and alterations of the cell proliferation and apoptosis mechanism, thus contributing to cancer development [3].

Myeloperoxidase (MPO) is an endogenous metabolic/oxidative lysosomal enzyme secreted by reactive neutrophils and monocytes. MPO plays an important role in carcinogenesis through activating procarcinogens to genotoxic intermediates and potentiation of xenobiotic carcinogenicity [4]. The MPO-463G > A polymorphism is located in the promoter region of MPO gene and is most extensively studied. This polymorphism may influence the MPO transcription level through removing a binding region for transcription factor Sp1, which can decrease the metabolic activation of carcinogenic compounds. Studies suggested that the MPO A allele carriers were associated with lower mRNA expression and transcription activity than the $463 \mathrm{G}$ common allele [5-7]. Abundant studies were conducted to investigate the role of this polymorphism in cancer development, including lung cancer, breast cancer, ovary cancer, gastric carcinoma and others, but results were inconsistent.

For instance, the association between MPO-463G > A polymorphism and lung cancer risk has attracted the most attention since the first meta-analysis performed in 2002 and suggested a slight protective effect of the MPO 463 A variant [8]. But research results in the following years varied a lot. Whether MPO-463G > A polymorphism could be a good predictor of cancer remained controversial. Up to 2010, a meta-analysis gave a systematic review into the association between MPO-463G > A polymorphism and cancer risk, including breast cancer, leukemia, and lung cancer [9]. This analysis did not report a significant association between these cancers and MPO-463G > A polymorphism, but suggested a moderately protective effect on cancer risk in Europeans. After that, many new studies attempted to further describe this association emerged, but came up with different results $[10,11]$.

In consideration of the recently published studies and the absence of a new systematic and comprehensive evaluation for the association between MPO-463G > A polymorphism and cancer risk, we conducted this metaanalysis to shed a light on this relationship.

\section{Methods}

\section{Search strategy}

Eligible literatures published before December 2015 were identified by searching PubMed, Cochrane Clinical Trials Database, Medline, EMBASE Google Scholar, and the Ovid Library with the following keywords: "Myeloperoxidase", "MPO", "polymorphism," or "variant" without any restriction on language. The scope of computerized literature search was expanded according to the reference lists of retrieved articles. The relevant original articles were also retrieved manually.

\section{Inclusion and exclusion criteria}

Any observational studies met the following criteria were included: (a) a study related to the MPO-463G > A polymorphism and cancer risk, (b) a case-control study, (c) the data of genotype frequency was available. Major reasons for exclusion of studies were as follows: (a) the use of only case-group data, (b) the duplication or overlap of previous publication, (c) the Newcastle-Ottawa Scale (NOS) score is less than 5 stars, and (d) familial type of cancer.

\section{Data extraction}

After removing duplicate studies, two investigators extracted the data individually from the reserved studies, and a third investigator was involved when discrepancies were raised. From each study, the following information was extracted: first author, journal, year of publication, country, ethnicity (Caucasian, Asian, and others), cancer type, source of controls (hospital-based studies, population-based studies), identification of cancer cases, genotyping methods, and the number of cases and controls for MPO-463G > A polymorphism.

\section{Quality assessment}

The Newcastle-Ottawa Scale (NOS) is a star rating system used to assess the study quality. The full score is defined as 9 stars, and 0 to 4 stars are usually considered to be a poor methodological quality while 5 to 9 stars are considered to be high quality. Any disagreements on the NOS score of these studies were solved by discussion and only studies with high quality were included.

\section{Statistical analysis}

The frequencies of the alleles and distributions of the genotypes of MPO-463G > A polymorphism in control groups were tested for Hardy-Weinberg equilibrium (HWE) using $X^{2}$ test. Odds ratios (OR) and 95\% confidence intervals $(95 \% \mathrm{CI})$ were used to assess the strength of associations between MPO-463G > A polymorphism and cancer risk. The combined ORs were calculated for dominant model (AA/GA versus GG), recessive model (AA versus $\mathrm{GA} / \mathrm{GG}$ ), additive model (AA versus $\mathrm{GG}$ ), 
and allele frequency model (A versus $G$ ) to assume the effects of the variant A allele, respectively. Stratified analyses were also conducted by cancer types, ethnicity, HWE, and source of controls.

The heterogeneity between studies was tested using the $Q$-statistic. $I^{2}$ metrics was used to determine the impact of heterogeneity and it was considered moderately when $I^{2}<50 \%$, and a fixed-effects model was utilized. Heterogeneity was considered statistically significant when $I^{2}>50 \%$ and a random-effects model was employed to calculate the pooled ORs [12]. Sensitivity analysis was carried out to evaluate the influence of individual study by excluding each study at a time. Publication bias was investigated with funnel plots and Egger's tests qualitatively. In addition, in order to evaluate the trend in OR over time, cumulative meta-analysis was performed. All statistical analyses were performed using STATA 11.2 statistical software.

\section{Results}

Literature search results and studies characteristics

After a comprehensive literature search, 1124 independent studies were preliminarily found. After excluding the uncorrelated and duplicate articles, 91 articles were included. Through reading the full texts, we further excluded 31 articles. Among them, 6 were not about MPO-463G > A polymorphisms, 11 had overlapping data, 4 did not report available data, 9 were metaanalyses, and 1 was not a case-control study. Therefore, the current meta-analysis included data from 60 articles that consisted of 38,614 subjects $(16,858$ cases and 21,756 controls). Figure 1 provides a summary of the selection process.

Table 1 shows a list of details of studies included in the current meta-analysis. The included articles comprised 25 lung cancer studies [8, 13-36], 5 breast cancer studies [37-41], 12 digestive system studies (gastrointestinal cancer: 3 esophagus studies, 3 stomach studies, and 2 colorectum studies; cancer of digestive organs: 3 liver studies, 1 pancreas studies) [42-53], 7 blood system studies (3 acute leukemia studies, 3 lymphoma studies, 1 multiple myeloma, and chronic granulocytic leukemia studies) [54-60] and 14 other studies ( 3 head and neck cancer studies, 3 gynecological tract cancer studies, 3 urological cancer studies, 2 musculoskeletal cancer studies, and 3 mix cancer studies) $[14,34,61-71]$. Cancers referred in the mix cancer studies included lung cancer, laryngeal cancer, and pharyngeal cancer [14]; lung cancer, prostate cancer [34]; multiple myeloma, and chronic granulocytic leukemia [58]. If the ethnicity was clearly described in the original studies, the studies would be categorized to the corresponding ethnicity group. If the original studies have not given a clear indication of ethnicity, the studies were categorized according to research region. If it has been specified in the original studies that the objects included were from different ethnicities, then studies should be categorized to the mix ethnicity group. Of the 60 studies, 24 were Asian background, 32 were Caucasian background, and 4 were mixed population background. Besides, the detailed description of hospital controls was addressed in Table 4 .

\section{Quantitative synthesis}

In total, significant association between MPO-463G > A polymorphism and cancer risk was observed under all the selected models when the eligible results were pooled together (Fig. 2). Results promoted that the mutant allele A and genotypes of AA as well as AA + GA might played protective roles in the development of cancer (AA versus $\mathrm{GG}$ : $\mathrm{OR}=0.84,95 \% \mathrm{CI}=0.76-0.94$; A versus $\mathrm{G}$ : $\mathrm{OR}=0.90,95 \% \mathrm{CI}=0.84-0.95$; $\mathrm{AA}$ versus $\mathrm{AG} /$ GG: $\mathrm{OR}=0.87,95 \% \mathrm{CI}=0.78-0.97$; $\mathrm{AA} / \mathrm{AG}$ versus $\mathrm{GG}$ : $\mathrm{OR}=0.89,95 \% \mathrm{CI}=0.83-0.95)$. The pooled results are summarized in Table 2.

\section{Stratified by cancer type}

According to the cancer type, when the minimum number of studies is greater than or equal to 5 , we combined the studies and conducted stratified analysis. Considering that gastrointestinal and digestive organs all belong to digestive system and might be affected by similar factors such as dietary factors, we grouped them together as digestive system cancer group. In the digestive system cancer group, significant association was seen in the allele frequency model $(\mathrm{OR}=0.71,95 \% \mathrm{CI}=0.57-0.88)$ and the dominant model $(\mathrm{OR}=0.67,95 \% \mathrm{CI}=0.53-0.84)$. In order to further discuss the cancer risk of $\mathrm{MPO}-463 \mathrm{G}>\mathrm{A}$ polymorphism, we divided digestive system cancer into digestive tract and digestive gland cancer. The results showed that allele A of MPO-463G $>$ A was significant with a decreased risk of digestive gland cancer in all the genetic models (A versus $\mathrm{G}: \mathrm{OR}=$ $0.63,95 \% \mathrm{CI}=0.40-0.99$; $\mathrm{AA}$ versus $\mathrm{GG}$ : $\mathrm{OR}=0.41$, $95 \% \mathrm{CI}=0.23-0.73 ; \quad \mathrm{AA}$ versus $\mathrm{AG} / \mathrm{GG}: \quad \mathrm{OR}=0.51$, $95 \% \mathrm{CI}=0.29-0.89 ; \quad \mathrm{AA} / \mathrm{AG}$ versus $\mathrm{GG}: \quad \mathrm{OR}=0.58$, $95 \% \mathrm{CI}=0.35-0.96$ ) and digestive tract cancers (A versus $\mathrm{G}: \mathrm{OR}=0.75,95 \% \mathrm{CI}=0.59-0.95 ; \mathrm{AA} / \mathrm{AG}$ versus GG:OR $=0.72,95 \% \mathrm{CI}=0.56-0.92$ ). Besides, in the lung cancer group, statistically significant finding was only observed in dominant model $(\mathrm{OR}=0.93,95 \% \mathrm{CI}$ $=0.87-0.99$ ). While, among studies of breast cancer and blood system cancer, no significant association was found in any genetic model. 


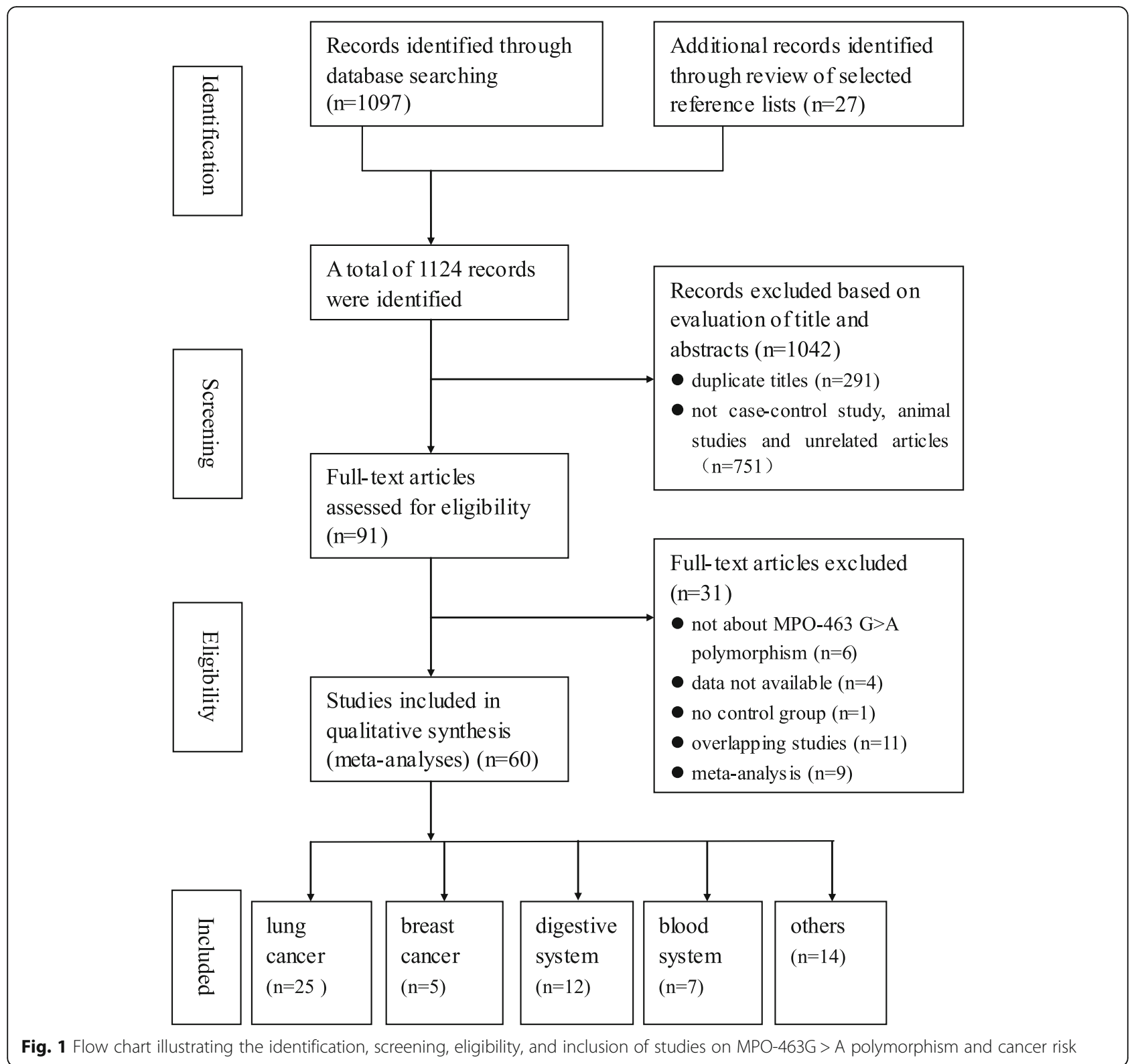

\section{Stratified by ethnicity}

In terms of ethnicity, we divided the ethnicity into three groups: Caucasian group, Asian group, and mix ethnicity group. The current result demonstrated that the genotype A may be a protect factor for both Caucasians and Asians, but not for mix ethnicity group. The different result from previous metaanalyses is seen in Table 3 .

When further stratified, the digestive system cancer subgroup by ethnicity, no significant association between MPO-463G > A polymorphism and Caucasians was found in any genetic model. Conversely, for the lung cancer subgroup, there was no significant association between MPO-463G $>$ A polymorphism and Asians (Table 3).

\section{Stratified by HWE}

Not all the studies included in this current analysis conformed to HWE. When stratified, in the HWE balanced group, the MPO-463G > A polymorphism was significantly associated with cancer risk in all the genetic models, while in the HWE un-balanced group, this association was only found in the recessive model and additive model. Results were represented in Table 2.

\section{Stratified by source of control group}

When it was stratified according to the source of control group, a statistically significant finding was found among hospital controls (A versus G: $\mathrm{OR}=0.892$, $95 \% \mathrm{CI}=0.811-0.981 ; \quad$ AA versus $\quad \mathrm{GG}: \quad \mathrm{OR}=0.79$, $95 \% \mathrm{CI}=0.68-0.93 ; \mathrm{AA} / \mathrm{AG}$ versus $\mathrm{GG}: \mathrm{OR}=0.88$, 
Table 1 Overview of studies included in the current meta-analysis

\begin{tabular}{|c|c|c|c|c|c|c|c|c|c|c|}
\hline Author & Year & Country & Ethics & Cancer & Disease confirmed & Resource & Case & Control & Genotyping & NOS score \\
\hline London & 1997 & America & mix Ethics & Lung cancer & NA & PB & 339 & 703 & RT-PCR & 7 \\
\hline Marchand & 2000 & America & mix Ethics & Lung cancer & Histological & PB & 323 & 437 & PCR-RFLP & 8 \\
\hline Misra & 2001 & Finland & Caucasian & Lung cancer & Histological & PB & 315 & 311 & PCR-RFLP & 8 \\
\hline $\mathrm{Xu}$ & 2002 & America & Caucasian & Lung cancer & Histological & $\mathrm{HB}$ & 989 & 1128 & PCR-RFLP & 7 \\
\hline LV & 2002 & China & Asian & Lung cancer & Histological & PB & 314 & 320 & PCR & 8 \\
\hline Kantarct & 2002 & America & Caucasian & Lung cancer & NA & $\mathrm{HB}$ & 307 & 307 & NA & 5 \\
\hline Feyler & 2002 & France & Caucasian & Lung cancer & Histological & $H B$ & 150 & 172 & capillary PCR & 7 \\
\hline Dally & 2002 & Germany & Caucasian & Lung cancer & NA & $\mathrm{HB}$ & 625 & 340 & PCR-RFLP. & 6 \\
\hline Chevriera & 2003 & France & Caucasian & Lung cancer & Histological & $\mathrm{HB}$ & 243 & 245 & PCR-RFLP & 7 \\
\hline Skuladottira & 2004 & Denmark & Caucasian & Lung cancer & NA & PB & 122 & 396 & NA & 6 \\
\hline Chana & 2004 & China & Asian & Lung cancer & NA & $\mathrm{HB}$ & 75 & 162 & PCR-RFLP & 6 \\
\hline Harms & 2004 & America & Caucasian & Lung cancer & Pathological & $\mathrm{PB}$ & 110 & 119 & MALDI-TOF & 8 \\
\hline Wu & 2004 & China & Asian & Lung cancer & Histological & PB & 98 & 112 & PCR & 8 \\
\hline Schabath & 2005 & America & Caucasian & Lung cancer & Histological & $\mathrm{HB}$ & 837 & 618 & PCR-RFLP & 7 \\
\hline Park & 2006 & Korea & Asian & Lung cancer & Histological & $\mathrm{HB}$ & 432 & 432 & PCR-RFLP & 7 \\
\hline Larsen & 2006 & Australia & Caucasian & Lung cancer & $\begin{array}{l}\text { Cytological } \\
\text { or histological }\end{array}$ & $\mathrm{HB}$ & 627 & 624 & PCR & 7 \\
\hline Yanga & 2007 & Korean & Asian & Lung cancer & Histological & $\mathrm{HB}$ & 318 & 353 & PCR-RFLP & 7 \\
\hline Zienolddiny & 2008 & Norway & Caucasian & Lung cancer & Pathologists & PB & 258 & 297 & NA & 7 \\
\hline Yoon & 2008 & Korea & Asian & Lung cancer & Histological & $\mathrm{HB}$ & 213 & 213 & Taqman probe & 7 \\
\hline Rotunno & 2009 & America & Caucasian & Lung cancer & Histology & PB & 185 & 2011 & NA & 7 \\
\hline Klinchid & 2009 & Thailand & Asian & Lung cancer & Histological & $\mathrm{HB}$ & 88 & 81 & PCR-RFLP & 7 \\
\hline Kiyohara & 2014 & Japan & Asian & Lung cancer & Histological & $\mathrm{HB}$ & 462 & 379 & TaqMan & 7 \\
\hline Bag & 2014 & India & Asian & Lung cancer & Tissue diagnosis & $\mathrm{HB}$ & 26 & 37 & PCR-RFLP & 7 \\
\hline Cascorbi & 2000 & Germany & Caucasian & Mix & NA & PB & 696 & 270 & PCR-RFLP & 7 \\
\hline Arslan & 2011 & Turkey & Caucasian & Mix & Histological & $\mathrm{HB}$ & 220 & 418 & PCR-RFLP & 7 \\
\hline Lin & 2004 & Taiwan & Asian & Breast cancer & $\begin{array}{l}\text { Medical charts } \\
\text { and pathology }\end{array}$ & PB & 99 & 366 & PCR-RFLP & 8 \\
\hline Ahn & 2004 & Canada & Caucasian & Breast cancer & NA & PB & 1011 & 1067 & PCR-RFLP. & 7 \\
\hline Yang & 2007 & America & Caucasian & Breast cancer & NA & PB & 406 & 392 & PCR-RFLP & 7 \\
\hline Li & 2009 & America & Caucasian & Breast cancer & NA & NA & 477 & 462 & PCR-RFLP. & 5 \\
\hline Tsai & 2012 & Taiwan & Asian & Breast cancer & NA & NA & 260 & 224 & PCR-RFLP & 5 \\
\hline Zhang & 2007 & China & Asian & Acute leukemia & $\begin{array}{l}\text { French-American-British } \\
\text { criteria }\end{array}$ & $\mathrm{HB}$ & 135 & 187 & Taqman & 7 \\
\hline Krajinovic & 2002 & Canada & Caucasian & ALL & Hematology-oncology & $\mathrm{HB}$ & 169 & 337 & PCR-RFLP & 7 \\
\hline Silveira & 2010 & Brazil & mix Ethics & ALL & Immunophenotyping & NA & 124 & 300 & PCR-RFLP & 6 \\
\hline Matsuo & 2001 & Japan & Asian & Lymphoma & Histological & $\mathrm{HB}$ & 372 & 241 & PCR-RFLP & 7 \\
\hline Saygilii & 2009 & Turkey & Caucasian & Mix & NA & $\mathrm{HB}$ & 62 & 40 & TaqMan & 6 \\
\hline Wang & 2006 & America & Caucasian & $\mathrm{NHL}$ & Histopathology & PB & 1082 & 905 & NA & 7 \\
\hline Farawela & 2012 & Egypt & Asian & $\mathrm{NHL}$ & Biopsy & $\mathrm{HB}$ & 100 & 100 & RT-PCR & 7 \\
\hline Funke & 2009 & Germany & Caucasian & Colorectal cancer & NA & PB & 627 & 603 & PCR & 7 \\
\hline Li & 2011 & China & Asian & Colorectal cancer & Histological & $\mathrm{HB}$ & 325 & 345 & PCR-RFLP & 7 \\
\hline Matsuo & 2001 & Japan & Asian & Esophageal cancer & NA & $\mathrm{HB}$ & 91 & 241 & PCR-RFLP & 6 \\
\hline $\mathrm{Li}$ & 2008 & China & Asian & Esophageal cancer & Pathologists & PB & 126 & 169 & PCR-RFLP & 8 \\
\hline $\mathrm{Li}$ & 2011 & China & Asian & Esophageal cancer & Histopathologic & $\mathrm{HB}$ & 94 & 280 & NA & 6 \\
\hline
\end{tabular}


Table 1 Overview of studies included in the current meta-analysis (Continued)

\begin{tabular}{|c|c|c|c|c|c|c|c|c|c|c|}
\hline Zhu & 2006 & China & Asian & Gastric cancer & $\begin{array}{l}\text { Biopsy or surgical } \\
\text { specimens }\end{array}$ & NA & 127 & 139 & PCR-RFLP & 6 \\
\hline Wang & 2011 & China & Asian & Gastric cancer & Pathological & $\mathrm{HB}$ & 62 & 61 & ASP-PCR & 7 \\
\hline Jang & 2012 & China & Asian & Gastric cancer & Pathological & $\mathrm{HB}$ & 117 & 105 & PCR-RFLP & 7 \\
\hline Pakakasama & 2003 & America & Caucasian & Hepatoblastoma & PCR-SSCP & PB & 48 & 180 & Pyrosequencing & 7 \\
\hline Nahon & 2011 & France & Caucasian & Hepatocellular cancer & Barcelona criteria & $H B$ & 84 & 121 & NA & 6 \\
\hline Carmo & 2012 & Brazil & Caucasian & Hepatocellular cancer & AASLD guidelines & $\mathrm{HB}$ & 32 & 252 & PCR-amplified & 7 \\
\hline Mustea & 2007 & Germany & Caucasian & Cervical cancer & Histological & $H B$ & 149 & 126 & PCR-RFLP & 7 \\
\hline Olson & 2004 & America & Caucasian & Ovarian cancer & NA & PB & 122 & 179 & TaqMan & 7 \\
\hline CastilloTong & 2014 & Austria & Caucasian & Ovarian cancer & Histopathological & NA & 305 & 299 & TaqMan probes & 6 \\
\hline Hung & 2004 & Italy & Caucasian & Bladder cancer & Histological & $\mathrm{HB}$ & 201 & 214 & PCR-RFLP & 7 \\
\hline Hsieh & 2010 & Taiwan & Asian & Leiomyoma & Pathological & $H B$ & 158 & 156 & PCR-RFLP & 7 \\
\hline Guo & 2010 & China & Asian & $\begin{array}{l}\text { Nasopharyngeal } \\
\text { carcinoma }\end{array}$ & Biopsy & $\mathrm{HB}$ & 358 & 629 & PCR-RFLP & 7 \\
\hline Wu & 2010 & Taiwan & Asian & Oral cavity & Pathological & $\mathrm{HB}$ & 122 & 122 & PCR & 7 \\
\hline Oliveira & 2007 & Brazil & mix Ethics & Osteosarcoma & NA & $\mathrm{HB}$ & 78 & 157 & PCR-RFLP & 6 \\
\hline Price & 2008 & Canada & Caucasian & Pancreatic cancer & Histological & $\mathrm{HB}$ & 122 & 331 & PCR & 7 \\
\hline Buch & 2008 & America & Caucasian & $\begin{array}{l}\text { Squamous } \\
\text { cell carcinoma }\end{array}$ & Biopsy-verified & PB & 193 & 414 & PCR-RFLP & 8 \\
\hline Choi & 2008 & America & Caucasian & Prostate cancer & Pathology & $\mathrm{HB}$ & 493 & 1332 & NA & 6 \\
\hline Tefik & 2013 & Turkey & Caucasian & Prostate cancer & Histological & $\mathrm{HB}$ & 155 & 195 & PCR & 7 \\
\hline
\end{tabular}

$N A$ not available, $A L L$ acute lymphoblastic leukemia, NAL non-Hodgkin lymphoma, mix: 2 or more cancer type, $P B$ population base, $H B$ hospital base, $P C R-R F L P$ polymerase chain reaction restriction fragment length polymorphism, $R T-P C R$ real-time polymerase chain reaction

$95 \% \mathrm{CI}=0.80-0.97 ; \quad$ AA versus $\quad \mathrm{AG} / \mathrm{GG}: \quad \mathrm{OR}=0.83$, $95 \% \mathrm{CI}=0.71-0.96)$, but no statistically significant evidence was found in controls based on population under most of the selected gene models except allele frequency model $\quad(\mathrm{OR}=0.91, \quad 95 \% \mathrm{CI}=0.83-0.97)$ (Table 4).

\section{Cumulative meta-analysis}

We performed cumulative meta-analyses for both overall cancer risk and lung cancer risk. For the former, as was shown in Fig. 3, the cumulative result of allele contrast showed a declining trend in the estimated protective effect in the vicinity of 0.890 during 2001 and 2014. And the relative change in the random-effects ORs was lower than 1.0 since 2001. Further, the increasingly narrower 95\%CIs suggested the precision of the estimates was gradually improved by continually adding more samples. In the same gene model, the inclination toward a non-significant association with overall lung cancer risk except for adding data provided by Heike in 2002 and Isabelle in 2003.

\section{Sensitivity analyses and publication bias}

Each included study was deleted one by one to reflect the influence of the individual data on the pooled ORs, and the corresponding results were not significantly changed. Moreover, there was no influence of publication bias in our study by using Begg's test or Egger's tests.

\section{Discussion}

The current analysis provided the most comprehensive investigation of MPO-463G > A polymorphism and cancer risk. Sixty studies consisted of 16,858 cases and 21,756 controls gave greater information to explore the association; however, the previous meta-analysis published in 2010 included only 43 studies with 14,171 cancer cases and 17,319 controls. The methodology used for this meta-analysis and the statistical evaluation of the results were well-developed. This meta-analysis demonstrated a new subgroup (digestive system cancer group) to discussed the association between MPO-463G $>$ A polymorphism and cancer risk in depth. In addition, we further stratified the digestive system cancer and lung cancer group by ethnicity and found some interesting results that the protect effect of MPO-463G > A polymorphism was only found in Caucasians in lung cancer population and Asians in digestive system cancer population. Furthermore, the cumulative meta-analysis which had not been conducted in previous relevant meta- 


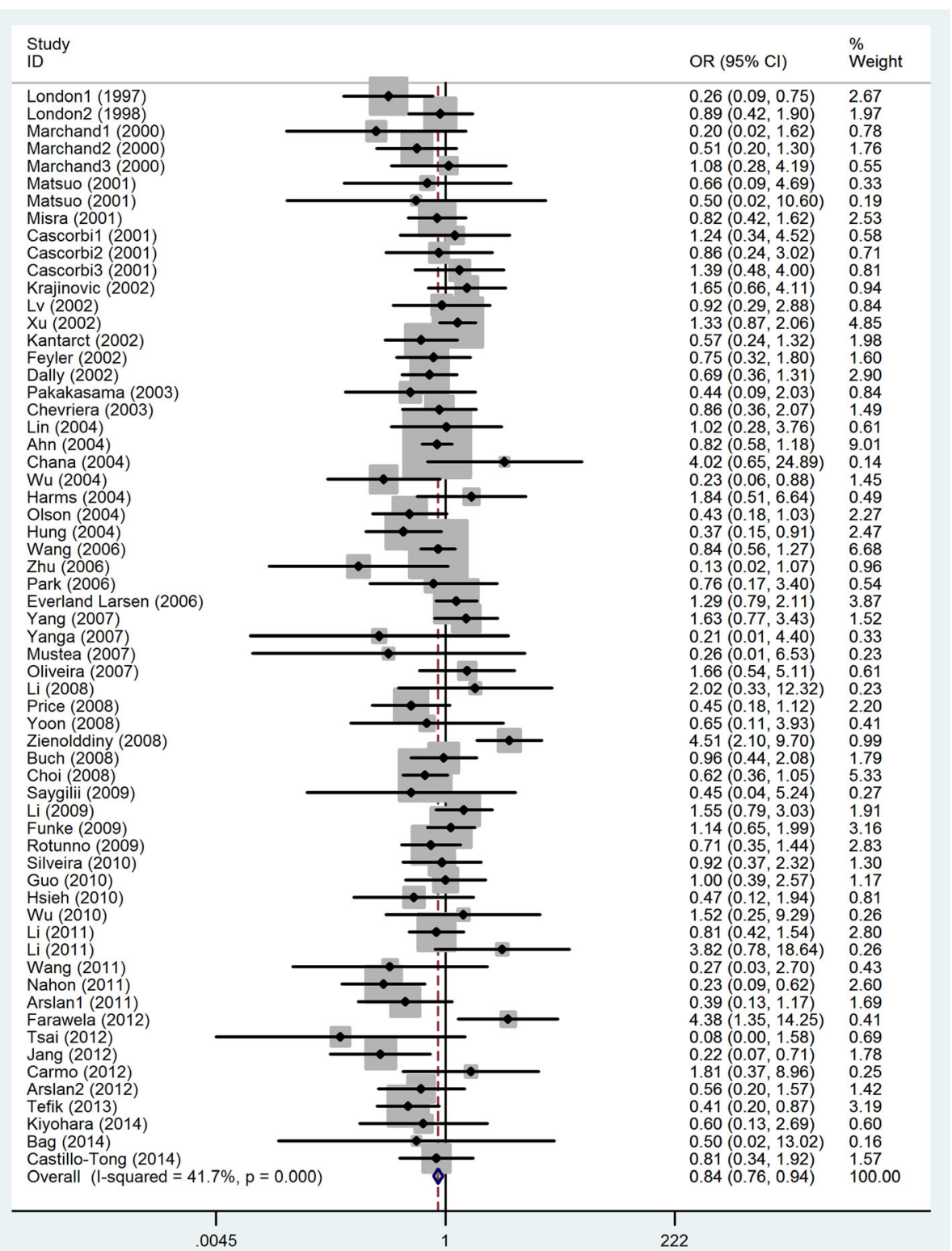

Fig. 2 Forest plots of meta-analysis of MPO-463G > A polymorphism in association with cancer risk (AA vs. GG)

Table 2 Stratified analyses of the MPO-463G > A polymorphism on cancer risk by cancer type

\begin{tabular}{|c|c|c|c|c|c|c|c|c|}
\hline \multirow{2}{*}{$\begin{array}{l}\text { Study } \\
\text { groups }\end{array}$} & \multicolumn{2}{|l|}{ A vs. G } & \multicolumn{2}{|l|}{ AA vs. GG } & \multicolumn{2}{|l|}{$A A$ vs. $A G+G G$} & \multicolumn{2}{|l|}{$\mathrm{AA}+\mathrm{AG}$ vs. GG } \\
\hline & $\overline{\mathrm{OR}}(95 \% \mathrm{Cl})$ & $R^{2}$ & $\overline{\mathrm{OR}}(95 \% \mathrm{Cl})$ & $R^{2}$ & $\overline{\mathrm{OR}}(95 \% \mathrm{Cl})$ & $1^{2}$ & $\overline{\mathrm{OR}}(95 \% \mathrm{Cl})$ & $R^{2}$ \\
\hline Total & $0.90(0.84-0.95)$ & 0.571 & $0.84(0.76-0.94)$ & 0.417 & $0.87(0.78-0.97)$ & 0.366 & $0.89(0.83-0.95)$ & 0.520 \\
\hline Lung & $0.92(0.83-1.01)$ & 0.525 & 0.93(0.78-1.10) & 0.481 & $0.95(0.80-1.12)$ & 0.488 & $0.93(0.87-0.99)$ & 0.420 \\
\hline Breast & $0.98(0.83-1.16)$ & 0.545 & $0.99(0.75-1.30)$ & 0.441 & $1.00(0.76-1.32)$ & 0.342 & $0.96(0.85-1.08)$ & 0.418 \\
\hline Digestive & $0.71(0.57-0.87)$ & 0.694 & 0.63(0.37-1.05) & 0.529 & $0.77(0.57-1.02)$ & 0.415 & $0.67(0.53-0.84)$ & 0.619 \\
\hline Dig tract & $0.75(0.59-0.95)$ & 0.668 & $0.74(0.39-1.43)$ & 0.523 & $0.92(0.65-1.29)$ & 0.449 & $0.72(0.56-0.92)$ & 0.577 \\
\hline Dig gland & $0.63(0.40-0.98)$ & 0.700 & $0.41(0.23-0.72)$ & 0.355 & $0.51(0.29-0.89)$ & 0.000 & $0.58(0.35-0.96)$ & 0.649 \\
\hline Blood & $1.12(0.96-1.25)$ & 0.431 & $0.96(0.63-1.58)$ & 0.501 & $1.03(0.85-1.40)$ & 0.623 & $1.03(0.85-1.40)$ & 0.623 \\
\hline
\end{tabular}


Table 3 Stratified analyses of the MPO-463G > A polymorphism on cancer risk by ethnicity and source of control group

\begin{tabular}{|c|c|c|c|c|c|c|c|c|}
\hline \multirow[t]{2}{*}{ Variables } & \multicolumn{2}{|l|}{ A vs. G } & \multicolumn{2}{|l|}{ AA vs. GG } & \multicolumn{2}{|l|}{$A A$ vs. $A G+G G$} & \multicolumn{2}{|l|}{$A A+A G$ vs. GG } \\
\hline & OR $(95 \% \mathrm{Cl})$ & $P^{2}$ & OR $(95 \% \mathrm{Cl})$ & $P^{2}$ & OR $(95 \% \mathrm{Cl})$ & $P^{2}$ & OR $(95 \% \mathrm{Cl})$ & $P^{2}$ \\
\hline Total & $0.90(0.854-1.069)$ & 0.571 & $0.84(0.76-0.94)$ & 0.417 & $0.89(0.83-0.95)$ & 0.520 & $0.87(0.78-0.97)$ & 0.366 \\
\hline \multicolumn{9}{|l|}{ Ethnicity } \\
\hline Caucasian & $0.90(0.84-0.97)$ & 0.576 & $0.85(0.76-0.96)$ & 0.506 & $0.91(0.84-0.98)$ & 0.533 & $0.87(0.78-0.98)$ & 0.484 \\
\hline Asian & $0.86(0.75-0.98)$ & 0.597 & $0.75(0.57-0.98)$ & 0.353 & $0.84(0.73-0.96)$ & 0.516 & $0.80(0.61-1.05)$ & 0.242 \\
\hline Other & $1.05(0.86-1.27)$ & 0.000 & $1.03(0.64-1.67)$ & 0.000 & $1.08(0.85-1.37)$ & 0.000 & $0.98(0.61-1.57)$ & 0.000 \\
\hline \multicolumn{9}{|l|}{ Lung cancer } \\
\hline Caucasian & $0.956(0.84-0.97)$ & 0.524 & $1.082(0.888-1.319)$ & 0.573 & 1.050(0.749-1.472) & 0.593 & $0.950(0.878-1.029)$ & 0.420 \\
\hline Asian & 0.863(0.691-1.078) & 0.551 & $0.641(0.370-1.110)$ & 0.013 & $0.709(0.400-1.256)$ & 0.000 & $0.861(0.740-1.002)$ & 0.495 \\
\hline Other & $0.861(0.715-1.037)$ & 0.176 & $0.567(0.363-0.886)$ & 0.000 & $0.558(0.360-0.867)$ & 0.000 & $0.926(0.758-1.132)$ & 0.467 \\
\hline \multicolumn{9}{|c|}{ Digestive Cancer } \\
\hline Caucasian & $0.704(0.474-1.045)$ & 0.796 & $0.609(0.291-1.274)$ & 0.620 & $0.751(0.514-1.100)$ & 0.428 & $0.658(0.423-1.024)$ & 0.756 \\
\hline Asian & $0.706(0.537-0.928)$ & 0.613 & $0.640(0.271-1.512)$ & 0.533 & $0.785(0.507-1.215)$ & 0.491 & $0.669(0.512-0.875)$ & 0.474 \\
\hline \multicolumn{9}{|l|}{ Resource } \\
\hline Population & $0.91(0.83-0.99)$ & 0.541 & $0.90(0.77-1.05)$ & 0.453 & $0.91(0.81-1.01)$ & 0.518 & $0.91(0.78-1.06)$ & 0.461 \\
\hline Hospital & $0.89(0.81-0.98)$ & 0.603 & $0.79(0.68-0.93)$ & 0.400 & $0.88(0.80-0.98)$ & 0.543 & $0.83(0.71-0.96)$ & 0.301 \\
\hline Others & $0.86(0.69-1.08)$ & 0.625 & $0.87(0.57-1.33)$ & 0.525 & $0.86(0.68-1.08)$ & 0.503 & $0.90(0.59-1.36)$ & 0.111 \\
\hline \multicolumn{9}{|l|}{ HWE } \\
\hline Yes & $0.89(0.83-0.96)$ & 0.598 & $0.88(0.77-0.96)$ & 0.454 & $0.88(0.81-0.95)$ & 0.507 & $0.92(0.81-1.04)$ & 0.373 \\
\hline No & $0.91(0.81-1.02)$ & 0.434 & $0.75(0.61-0.93)$ & 0.167 & 0.93(0.79-1.10) & 0.607 & $0.74(0.60-0.91)$ & 0.297 \\
\hline
\end{tabular}

analysis provided more powerful evidence that the results were reliable. Moreover, NOS was used to evaluate the quality of the studies, and results suggested that all the studies included in the current meta-analysis were high quality.

Contrary to the earlier meta-analysis which indicated that no significant association was found in any genetic model, the current meta-analysis showed evidence that allele A was associated with a reduced cancer risk compared with $G$ allele. Besides, in view of the cumulative analysis results, we intended to draw a conclusion that MPO-463G > A polymorphism had a protective significance of cancer risk. But when the studies were stratified by cancer type, ethnicity, HWE, and control resource, results differed.

Among all the cancers studied in our meta-analysis, lung cancer was mostly discussed. We were interested to find that the results of three related meta-analyses published in 2013, were not identical with each other. Zhou YY et al. found MPO-463G > A polymorphism was significantly associated with decreased risk of lung cancer risk in Asians under additive model and recessive model [72]. Zhou $\mathrm{C}$ et al. suggested that in allele frequency and dominant models, when stratified by ethnicity, evidence showed a protect effect in Caucasians, but not in Asians [73]. While, Yang et al. indicated there was no significant association of both overall and stratified analyses according to ethnicity, source of controls and smoking status [74]. What is more, another two metaanalyses published in 2014 by $\mathrm{Li}$ and Huang et al. $[75,76]$, respectively, also got different conclusions. The varied results may have relation with the different search strategies, selection criteria, quality of the original studies and so on. Considering the confusing outcome of the lung cancer, we further calculated the data and tried to come to a convincing conclusion. We considered our results more reliable, for all the studies included were of high quality and we removed the duplicated data provided by $\mathrm{Li}$ and Huang et al. $[75,76]$. As described above, the cumulative result provided a more powerful evidence and we intended to draw a conclusion that MPO-463G > A polymorphism may not be a good predictor of lung cancer both in Caucasians or Asians.

We first conducted a digestive system cancer group in meta-analysis and found significant results in allele frequency and dominant models. When it was divided into digestive tract and digestive gland, results indicated that digestive gland was more closely linked with decreasing cancer risk. Similarly, Samart et al. [43] even found that A allele and G/A or A/A separately reduced the risk of hepatoblastoma of 50 and $56 \%$ in Caucasians. Our analysis demonstrated that A allele and AG/AA had a 0.71fold cancer risk and 0.67 -fold cancer risk separately. 
Table 4 The hospital control's details

\begin{tabular}{|c|c|c|}
\hline Author & Year & details \\
\hline Matsuo & 2001 & Outpatients without any history of cancer \\
\hline Matsuo & 2001 & Non-cancer controls \\
\hline $\mathrm{Xu}$ & 2002 & Friends or spouses of patients (with either lung cancer or other cardiothoracic problems), with no matching characteristics \\
\hline Krajinovic & 2002 & $\begin{array}{l}\text { Selected from a large institutional DNA bank. Care was taken to match the patient population by selecting controls } \\
\text { of French-Canadian origin served by Sainte-Justine Hospital }\end{array}$ \\
\hline Kantarct & 2002 & Without diagnosis of lung cancer \\
\hline Feyler & 2002 & $\begin{array}{l}\text { Frequency matched on age, sex, and hospital, consisted of all consecutive Caucasian patients without previous or current } \\
\text { malignant diseases }\end{array}$ \\
\hline Dally & 2002 & $\begin{array}{l}\text { Had no previous or present history of malignant diseases: the main diagnoses included alveolitis, bronchitis,pneumonia, } \\
\text { fibrosis,sarcoidosis, COPD and emphysema }\end{array}$ \\
\hline Chevriera & 2001 & All subjects hospitalized for different disorders except cancer \\
\hline Chana & 2004 & $\begin{array}{l}\text { Had no history of pulmonary diseases, and were receiving health evaluation for other reasons and matched for sex } \\
\text { and age with the lung cancer patients }\end{array}$ \\
\hline Hung & 2004 & $\begin{array}{l}\text { Patients admitted to the same hospitals during the same period of time, with urological non-neoplastic diseases, } \\
\text { including hydronephrosis, urolithiasis, malformative urological diseases, prostatic adenoma, and hypertrophia, } \\
\text { urological traumas, orchiepididymitis, hydrocele and unspecified urinary symptoms }\end{array}$ \\
\hline Schabath & 2005 & $\begin{array}{l}\text { Healthy controls frequency matched to the cases on age ( } \pm 5 \text { years), gender,ethnicity, and smoking status } \\
\text { (current, former, and never) }\end{array}$ \\
\hline Park & 2006 & Healthy volunteers \\
\hline Larsen & 2006 & $\begin{array}{l}\text { Controls consisted of patients with chronic obstructive pulmonary disease (COPD) but without lung cancer ( } n \text { 1/4 380), } \\
\text { treated at the same hospital from } 1998 \text { to 2003, or healthy smokers attending a smoking cessation clinic held at the } \\
\text { hospital from } 2000 \text { to } 2003\end{array}$ \\
\hline Zhang & 2007 & No known malignant diseases \\
\hline Yanga & 2007 & Healty individuals without lung cancer or any other cancer \\
\hline Oliveira & 2007 & Individuals admitted in the Pediatric department of the Federal University of Sao Paulo, Brazil without osteosarcoma \\
\hline Mustea & 2007 & $\begin{array}{l}\text { The control group consisted of similarly aged women with no history of cancer and all of them were treated for benign } \\
\text { gynecological diseases. None of them had previously undergone a hysterectomy }\end{array}$ \\
\hline Yoon & 2008 & Healthy control \\
\hline Price & 2008 & $\begin{array}{l}\text { Healthy controls were frequency matched for age and sex. The controls were healthy nonblood-related family members } \\
\text { (usually spouses) and friends of other cancer/surgical patients and were used as a shared set of controls for aerodigestive cancers }\end{array}$ \\
\hline Choi & 2008 & Free of both prostate cancer and lung cancer \\
\hline Saygilii & 2009 & Healthy volunteers and No one in the control group had a smoking history or chronic use of any drugs \\
\hline Klinchid & 2009 & Healthy volunteers and diabetic patients \\
\hline Wu & 2010 & With the same habits and without a present or previous history of any cancer \\
\hline Hsieh & 2010 & Non-leiomyoma \\
\hline Guo & 2010 & $\begin{array}{l}\text { Spouse or geographically matched residents who were EBV/IgANCA positive (IgA+) or EBV/IgA/ VCA negative (IgA-) } \\
\text { and NPC free at the time of study enrollment }\end{array}$ \\
\hline Nahon & 2011 & HCV-induced cirrhosis \\
\hline Wang & 2011 & Non-cancer controls \\
\hline Li & 2011 & Healthy had no current or previous diagnosis of cancer and genetic disease \\
\hline $\mathrm{Li}$ & 2011 & Diagnosed as normal by histopathology of ophageal squamous epithelial cells \\
\hline Arslan & 2011 & Healthy individuals without any history of cancer \\
\hline Jang & 2012 & Individuals without gastic cancer \\
\hline Carmo & 2012 & $\begin{array}{l}\text { They had persistent anti-HCV antibodies and were HCVRNA positive. Presence of hepatitis A, hepatitis B, } \\
\text { and immunodeficiency virus (HIV) antibodies were considered as exclusion criteria }\end{array}$ \\
\hline Tefik & 2013 & Normal DRE and serum PSA levels of $<4 \mathrm{ng} / \mathrm{mL}$ \\
\hline Bag & 2014 & Normal healthy individuals with no history of cancer \\
\hline Kiyohara & 2014 & Without a clinical history of any type of cancer past or present, ischemic heart disease or chronic respiratory diseases \\
\hline
\end{tabular}




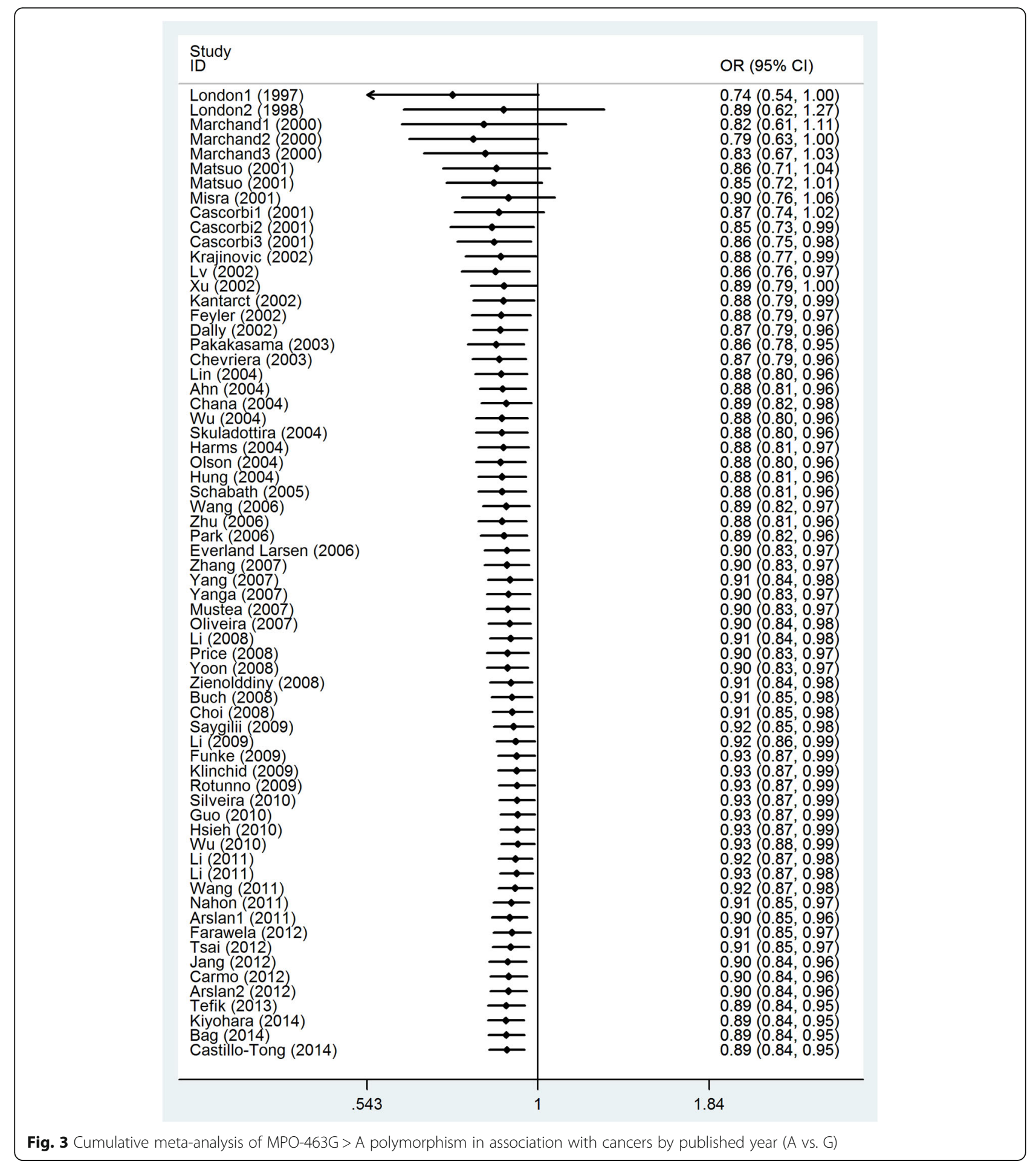

As for breast cancer, though positive result had been published before [11], whether it had a relationship with high level of MPO-G463 > A polymorphism stayed confusing. Our result for breast cancer was in line with the result by $\mathrm{Chu}$ et al. [9], suggesting a non-significant association. In addition, giving to the fact the level of MPO-containing neutrophils were high in breast tissue with or without cancer [77, 78], we tend to believe that MPO-463G > A polymorphism could not predict breast cancer well. A similar situation was seen in the blood system group, and the current study suggested no significant association between it and MPO-463G > A polymorphism. 
Regarding the source of controls, the results should also be concerned. Only in the hospital population, significant results could be observed. This might reflect influence of health status, gene-gene or gene-environment interactions. Well-matched controls should be included in the future studies. Ethnicity is often one of the sources of heterogeneity. Stratifying the ethnicity, strength was observed in Caucasians and Asians under almost all the genetic models instead of the mix ethnicity group. Meanwhile, no significant association between MPO-463G $>$ A polymorphism and digestive system cancer for Caucasians or lung cancer for Asians were found in any genetic model. Accordingly, we suggest the main source of heterogeneity is from the ethnicities, source of controls, and different cancer itself. And it may have something to do with age, sex, sample size, ethnic background, gene-gene interaction, environment background, and lifestyle. So it is meaningful to discuss this association more detailed. For example, analyses striated by age, sex, and accumulation of sample size are considerable.

Limited data of digestive system cancer and blood system cancer are one of the deficiencies of our metaanalysis. In addition, gene-gene interactions, environment background, and lifestyle were not well addressed in the meta-analysis for the lack of data. Taking it into consideration, more studies aimed to discuss the relationship of MPO-463G > A polymorphism and digestive system cancer, blood system cancer or the gene-gene or gene-environment interactions should be conducted to give a more deeper knowledge of this association. Besides, the lack of original information about age also limited our more in-depth research. We hope future researches can provide detailed information about age and then the stratified analysis by age could be done.

\section{Conclusion}

Overall, in cumulative analysis, the stable trend indicated that evidence was sufficient to show the association between MPO-463G > A polymorphism and cancer risk. MPO-463G > A polymorphism might have an effect in reducing the risk of digestive system cancer, but might not be a good predictor of lung cancer, breast cancer, and blood system cancers. It was significantly associated with cancer risk both in Caucasians and Asians. But no significant association between MPO-463G $>$ A polymorphism and Caucasians was found in any genetic model for digestive system cancer and no significant association between MPO-463G > A polymorphism and Asians was found for lung cancer. More studies exploring the association between MPO-463G $>$ A polymorphism and digestive and blood system cancer are needed in the future.
Acknowledgements

Not applicable.

Availability of data and materials

Please contact author for data requests.

\section{Funding}

This study was partially supported by research funding from the National Natural Science Foundation (No. 81160298). The funders had no role in study design, data collection and analysis, decision to publish, or preparation of the manuscript.

\section{Author contributions}

W-jY, M-yW, F-ZP, and HC conceived and designed the experiments. W-jY and M-yW performed the experiments. F-zP analyzed the data. W-jY, M-yW, F-ZP, and $\mathrm{HC}$ wrote the paper. All authors read and approved the final manuscript.

Ethics approval and consent to participate

Not applicable.

Consent for publication

Not applicable.

Competing interests

The authors declare that they have no competing interests.

\section{Publisher's Note}

Springer Nature remains neutral with regard to jurisdictional claims in published maps and institutional affiliations.

\section{Author details}

1Department of Gastroenterology, The Second Affiliated Hospital of Guilin Medical University, Gui Lin 541100, China. ${ }^{2}$ Department of Chemotherapy, Affiliated Tumor Hospital of Guangxi Medical University, Nanning 530021, Guangxi, China. ${ }^{3}$ Department of Cardiology, The First Affiliated Hospital of Guangxi Medical University, Nanning 530021, China. ${ }^{4}$ Department of Gastroenterology, The First Affiliated Hospital of Guangxi Medical University, Nanning 530021, China.

Received: 11 October 2016 Accepted: 22 June 2017

Published online: 02 August 2017

References

1. Pharoah PD, Dunning AM, Ponder BA, Easton DF. Association studies for finding cancer-susceptibility genetic variants. Nat Rev Cancer. 2004;4(11):850-60.

2. Ghodbane S, Lahbib A, Sakly M, Abdelmelek H. Bioeffects of static magnetic fields: oxidative stress, genotoxic effects, and cancer studies. Biomed Res Int. 2013;2013:602987

3. Steenport M, Eom H, Uezu M, Schneller J, Gupta R, Mustafa Y, Villanueva R, Straus EW, Raffaniello RD. Association of polymorphisms in myeloperoxidase and catalase genes with precancerous changes in the gastric mucosa of patients at inner-city hospitals in New York. Oncol Rep. 2007;18(1):235-40.

4. Klebanoff SJ. Myeloperoxidase. Proc Assoc Am Physicians. 1999;111(5):383-9.

5. Piedrafita FJ, Molander RB, Vansant G, Orlova EA, Pfahl M, Reynolds WF. An Alu element in the myeloperoxidase promoter contains a composite SP1-thyroid hormone-retinoic acid response element. J Biol Chem. 1996;271(24):14412-20.

6. Kumar AP, Piedrafita FJ, Reynolds WF. Peroxisome proliferator-activated receptor gamma ligands regulate myeloperoxidase expression in macrophages by an estrogen-dependent mechanism involving the -463GA promoter polymorphism. J Biol Chem. 2004;279(9):8300-15.

7. Winterbourn CC, Vissers MC, Kettle AJ. Myeloperoxidase. Curr Opin Hematol. 2000:7(1):53-8.

8. Feyler A, Voho A, Bouchardy C, Kuokkanen K, Dayer P, Hirvonen A, Benhamou S. Point: myeloperoxidase $-463 G$ - a polymorphism and lung cancer risk. Cancer epidemiology, biomarkers \& prevention : a publication of the American Association for Cancer Research, cosponsored by the American Society of Preventive Oncology. 2002;11(12):1550-4.

9. Chu H, Wang M, Wang M, Gu D, Wu D, Zhang Z, Tang J, Zhang Z. The MPO-463G > A polymorphism and cancer risk: a meta-analysis based on 43 case-control studies. Mutagenesis. 2010;25(4):389-95. 
10. Pabalan N, Jarjanazi $H$, Sung L, Li H, Ozcelik H. Menopausal status modifies breast cancer risk associated with the myeloperoxidase (MPO) G463A polymorphism in Caucasian women: a meta-analysis. PLoS One. 2012;7(3), e32389.

11. Qin X, Deng Y, Zeng ZY, Peng QL, Huang XL, Mo CJ, Li S, Zhao JM. Myeloperoxidase polymorphism, menopausal status, and breast cancer risk: an update meta-analysis. PLoS One. 2013;8(8), e72583.

12. Zintzaras E, Kitsios G, Stefanidis I. Endothelial NO synthase gene polymorphisms and hypertension: a meta-analysis. Hypertension. 2006;48(4):700-10.

13. London SJ, Lehman TA, Taylor JA. Myeloperoxidase genetic polymorphism and lung cancer risk. Cancer Res. 1997;57(22):5001-3.

14. Cascorbi I, Henning S, Brockmoller J, Gephart J, Meisel C, Muller JM, Loddenkemper $\mathrm{R}$, Roots I. Substantially reduced risk of cancer of the aerodigestive tract in subjects with variant $-463 \mathrm{~A}$ of the myeloperoxidase gene. Cancer Res. 2000;60(3):644-9.

15. Le Marchand L, Seifried A, Lum A, Wilkens LR. Association of the myeloperoxidase $-463 G$ - a polymorphism with lung cancer risk. Cancer epidemiology, biomarkers \& prevention : a publication of the American Association for Cancer Research, cosponsored by the American Society of Preventive Oncology. 2000;9(2):181-4.

16. Misra RR, Tangrea JA, Virtamo J, Ratnasinghe D, Andersen MR, Barrett M, Taylor PR, Albanes D. Variation in the promoter region of the myeloperoxidase gene is not directly related to lung cancer risk among male smokers in Finland. Cancer Lett. 2001;164(2):161-7.

17. Dally $H$, Gassner $K$, Jager B, Schmezer $P$, Spiegelhalder B, Edler L, Drings $P$, Dienemann H, Schulz V, Kayser $K$, et al. Myeloperoxidase (MPO) genotype and lung cancer histologic types: the MPO -463 A allele is associated with reduced risk for small cell lung cancer in smokers. International journal of cancer Journal international du cancer. 2002;102(5):530-5.

18. Kantarci $\mathrm{OH}$, Lesnick TG, Yang P, Meyer RL, Hebrink DD, McMurray CT, Weinshenker BG. Myeloperoxidase $-463(\mathrm{G}->\mathrm{A})$ polymorphism associated with lower risk of lung cancer. Mayo Clin Proc. 2002;77(1):17-22.

19. Lu W, Xing D, Qi J, Tan W, Miao X, Lin D. Genetic polymorphism in myeloperoxidase but not GSTM1 is associated with risk of lung squamous cell carcinoma in a Chinese population. International journal of cancer Journal international du cancer. 2002;102(3):275-9.

20. Xu LL, Liu G, Miller DP, Zhou W, Lynch TJ, Wain JC, Su L, Christiani DC Counterpoint: the myeloperoxidase $-463 \mathrm{G}$ - a polymorphism does not decrease lung cancer susceptibility in Caucasians. Cancer epidemiology, biomarkers \& prevention : a publication of the American Association for Cancer Research, cosponsored by the American Society of Preventive Oncology. 2002;11(12):1555-9.

21. Chevrier I, Stucker I, Houllier AM, Cenee S, Beaune P, Laurent-Puig P, Loriot MA. Myeloperoxidase: new polymorphisms and relation with lung cancer risk. Pharmacogenetics. 2003;13(12):729-39.

22. Wu XM, Zhou YK, Ren S, Hao QL. Gene polymorphism of myeloperoxidase and genetic susceptibility to lung cancer. Ai zheng = Aizheng $=$ Chinese journal of cancer. 2003;22(9):912-5.

23. Harms C, Salama SA, Sierra-Torres CH, Cajas-Salazar N, Au WW. Polymorphisms in DNA repair genes, chromosome aberrations, and lung cancer. Environ Mol Mutagen. 2004;44(1):74-82.

24. Chan EC, Lam SY, Fu KH, Kwong YL. Polymorphisms of the GSTM1, GSTP1, MPO, XRCC1, and NQO1 genes in Chinese patients with non-small cell lung cancers: relationship with aberrant promoter methylation of the CDKN2A and RARB genes. Cancer Genet Cytogenet. 2005;162(1):10-20.

25. Schabath MB, Delclos GL, Martynowicz MM, Greisinger AJ, Lu C, Wu X, Spitz MR. Opposing effects of emphysema, hay fever, and select genetic variants on lung cancer risk. Am J Epidemiol. 2005;161(5):412-22.

26. Skuladottir H, Autrup H, Autrup J, Tjoenneland A, Overvad K, Ryberg D, Haugen A, Olsen JH. Polymorphisms in genes involved in xenobiotic metabolism and lung cancer risk under the age of 60 years. A pooled study of lung cancer patients in Denmark and Norway. Lung cancer (Amsterdam, Netherlands). 2005;48(2):187-99.

27. Larsen JE, Colosimo ML, Yang IA, Bowman R, Zimmerman PV, Fong KM. CYP1A1 lle462Val and MPO G-463A interact to increase risk of adenocarcinoma but not squamous cell carcinoma of the lung. Carcinogenesis. 2006;27(3):525-32.

28. Park JH, Park JM, Kim EJ, Cha SI, Lee EB, Kim CH, Kam S, Jung TH, Park JY. Myeloperoxidase-463G > A polymorphism and risk of primary lung cancer in a Korean population. Cancer Detect Prev. 2006;30(3):257-61.
29. Yang M, Choi Y, Hwangbo B, Lee JS. Combined effects of genetic polymorphisms in six selected genes on lung cancer susceptibility. Lung cancer (Amsterdam, Netherlands). 2007;57(2):135-42.

30. Yoon KA, Kim JH, Gil HJ, Hwang H, Hwangbo B, Lee JS. CYP1B1, CYP1A1, MPO, and GSTP1 polymorphisms and lung cancer risk in never-smoking Korean women. Lung cancer (Amsterdam, Netherlands). 2008;60(1):40-6.

31. Zienolddiny S, Campa D, Lind H, Ryberg D, Skaug V, Stangeland LB, Canzian F, Haugen A. A comprehensive analysis of phase I and phase II metabolism gene polymorphisms and risk of non-small cell lung cancer in smokers. Carcinogenesis. 2008;29(6):1164-9.

32. Klinchid J, Chewaskulyoung B, Saeteng S, Lertprasertsuke N, Kasinrerk W, Cressey R. Effect of combined genetic polymorphisms on lung cancer risk in northern Thai women. Cancer Genet Cytogenet. 2009;195(2):143-9.

33. Rotunno M, Yu K, Lubin JH, Consonni D, Pesatori AC, Goldstein AM, Goldin LR, Wacholder S, Welch R, Burdette L, et al. Phase I metabolic genes and risk of lung cancer: multiple polymorphisms and mRNA expression. PLoS One. 2009;4(5), e5652.

34. Arslan S, Pinarbasi H, Silig Y. Myeloperoxidase G-463A polymorphism and risk of lung and prostate cancer in a Turkish population. Mol Med Rep. 2011:4(1):87-92.

35. Bag A, Bag N, Jeena LM, Jyala NS. Glutathione S-transferase T1 and myeloperoxidase $-463 \mathrm{G}>\mathrm{A}$ genotypes in lung cancer patients of Kumaun region. Obstet Gynecol Int. 2014;5(2):293-6.

36. Kiyohara C, Horiuchi T, Takayama K, Nakanishi Y. Genetic polymorphisms involved in the inflammatory response and lung cancer risk: a case-control study in Japan. Cytokine+. 2014;65(1):88-94.

37. Ahn J, Gammon MD, Santella RM, Gaudet MM, Britton JA, Teitelbaum SL, Terry MB, Neugut Al, Josephy PD, Ambrosone CB. Myeloperoxidase genotype, fruit and vegetable consumption, and breast cancer risk. Cancer Res. 2004;64(20):7634-9.

38. Lin SC, Chou YC, Wu MH, Wu CC, Lin WY, Yu CP, Yu JC, You SL, Chen CJ, Sun CA. Genetic variants of myeloperoxidase and catechol-Omethyltransferase and breast cancer risk. European journal of cancer prevention : the official journal of the European Cancer Prevention Organisation (ECP). 2005;14(3):257-61.

39. Yang J, Ambrosone CB, Hong CC, Ahn J, Rodriguez C, Thun MJ, Calle EE. Relationships between polymorphisms in NOS3 and MPO genes, cigarette smoking and risk of post-menopausal breast cancer. Carcinogenesis. 2007;28(6):1247-53.

40. Li Y, Ambrosone CB, McCullough MJ, Ahn J, Stevens VL, Thun MJ, Hong CC. Oxidative stress-related genotypes, fruit and vegetable consumption and breast cancer risk. Carcinogenesis. 2009;30(5):777-84.

41. Tsai SM, Wu SH, Hou MF, Chen YL, Ma H, Tsai LY. Oxidative stress-related enzyme gene polymorphisms and susceptibility to breast cancer in nonsmoking, non-alcohol-consuming Taiwanese women: a case-control study. Ann Clin Biochem. 2012:49(Pt 2):152-8.

42. Matsuo K, Hamajima N, Shinoda M, Hatooka S, Inoue M, Takezaki T, Onda H, Tajima K. Possible risk reduction in esophageal cancer associated with MPO -463 A allele. Journal of epidemiology / Japan Epidemiological Association. 2001:11(3):109-14

43. Pakakasama S, Chen TT, Frawley W, Muller C, Douglass EC, Tomlinson GE. Myeloperoxidase promotor polymorphism and risk of hepatoblastoma. International journal of cancer Journal international du cancer. 2003;106(2):205-7.

44. Zhu H, Yang L, Zhou B, Yu R, Tang N, Wang B. Myeloperoxidase G-463A polymorphism and the risk of gastric cancer: a case-control study. Carcinogenesis. 2006;27(12):2491-6.

45. Li D, Diao Y, Li H, Fang X, Li H. Association of the polymorphisms of MTHFR C677T, VDR C352T, and MPO G463A with risk for esophageal squamous cell dysplasia and carcinoma. Arch Med Res. 2008;39(6):594-600.

46. Wheatley-Price P, Asomaning K, Reid A, Zhai R, Su L, Zhou W, Zhu A, Ryan DP, Christiani DC, Liu G. Myeloperoxidase and superoxide dismutase polymorphisms are associated with an increased risk of developing pancreatic adenocarcinoma. Cancer. 2008;112(5):1037-42.

47. Funke $\mathrm{S}$, Hoffmeister $\mathrm{M}$, Brenner $\mathrm{H}$, Chang-Claude J. Effect modification by smoking on the association between genetic polymorphisms in oxidative stress genes and colorectal cancer risk. Cancer epidemiology, biomarkers \& prevention : a publication of the American Association for Cancer Research, cosponsored by the American Society of Preventive Oncology. 2009;18(8): 2336-8. 
48. Li QD, Li H, Wang MS, Diao TY, Zhou ZY, Fang QX, Yang FY, Li QH. Multisusceptibility genes associated with the risk of the development stages of esophageal squamous cell cancer in Feicheng County. BMC Gastroenterol. 2011;11:74.

49. Li Y, Qin Y, Wang ML, Zhu HF, Huang XE. The myeloperoxidase-463G > A polymorphism influences risk of colorectal cancer in southern China: a casecontrol study. Asian Pac J Cancer Prev. 2011;12(7):1789-93.

50. Wang Q, Yan ZQ, Wang HB, Xie HT, Yu Y. Analysis for myeloperoxidase genetic polymorphism in gastric cancer. Zhonghua wei chang wai ke za zhi $=$ Chinese journal of gastrointestinal surgery. 2011;14(7):542-4.

51. do Carmo RF, de Almeida DB, Aroucha DC, Vasconcelos LR, de Moraes AC, de Mendonca Cavalcanti Mdo S, de Morais CN, Pereira LM, Moura P. Plasma myeloperoxidase levels correlate with hepatocellular carcinoma in chronic hepatitis C. Hum Immunol. 2012;73(11):1127-31.

52. Jiang Z, Zhang Z, Wang Z, Zhang G, Hu K, He B, Wang S. Association of the 463G-A myeloperoxidase gene polymorphism with gastric cancer risk. Hepatogastroenterology. 2012;59(115):757-61.

53. Nahon $P$, Sutton A, Rufat $P$, Charnaux N, Mansouri A, Moreau R, Ganne-Carrie N, Grando-Lemaire V, N'Kontchou G, Trinchet JC, et al. A variant in myeloperoxidase promoter hastens the emergence of hepatocellular carcinoma in patients with HCV-related cirrhosis. J Hepatol. 2012;56(2):426-32

54. Matsuo K, Hamajima N, Suzuki R, Nakamura S, Seto M, Morishima Y, Tajima K. No substantial difference in genotype frequencies of interleukin and myeloperoxidase polymorphisms between malignant lymphoma patients and non-cancer controls. Haematologica. 2001;86(6):602-8.

55. Krajinovic M, Sinnett H, Richer C, Labuda D, Sinnett D. Role of NQO1, MPO and CYP2E1 genetic polymorphisms in the susceptibility to childhood acute lymphoblastic leukemia. International journal of cancer Journal international du cancer. 2002:97(2):230-6.

56. Wang SS, Davis S, Cerhan JR, Hartge P, Severson RK, Cozen W, Lan Q, Welch R, Chanock SJ, Rothman N. Polymorphisms in oxidative stress genes and risk for non-Hodgkin lymphoma. Carcinogenesis. 2006;27(9):1828-34.

57. Zhang J, Zhu FY, Pu YP, Yin LH, Luo J, Wang WP, Zhou GH. Analysis of multiple single nucleotide polymorphisms (SNPs) of myeloperoxidase (MPO) to screen for genetic markers associated with acute leukemia in Chinese Han population. J Toxicol Environ Health A. 2007;70(11):901-7.

58. Saygili El, Aksoy N, Pehlivan M, Sever T, Yilmaz M, Cimenci IG, Pehlivan S. Enzyme levels and G-463A polymorphism of myeloperoxidase in chronic lymphocytic leukemia and multiple myeloma. Leuk Lymphoma. 2009;50(12):2030-7.

59. Silveira Vda S, Canalle R, Scrideli CA, Queiroz RG, Tone LG. Role of the CYP2D6, EPHX1, MPO, and NQO1 genes in the susceptibility to acute lymphoblastic leukemia in Brazilian children. Environ Mol Mutagen. 2010; 51(1):48-56.

60. Farawela H, Khorshied M, Shaheen I, Gouda H, Nasef A, Abulata N, Mahmoud HA, Zawam HM, Mousa SM. The association between hepatitis C virus infection, genetic polymorphisms of oxidative stress genes and B-cel non-Hodgkin's lymphoma risk in Egypt. Infection, genetics and evolution : journal of molecular epidemiology and evolutionary genetics in infectious diseases. 2012;12(6):1189-94.

61. Hung RJ, Boffetta P, Brennan P, Malaveille C, Gelatti U, Placidi D, Carta A, Hautefeuille A, Porru S. Genetic polymorphisms of MPO, COMT, MnSOD, NQO1, interactions with environmental exposures and bladder cancer risk. Carcinogenesis. 2004;25(6):973-8.

62. Olson SH, Carlson MD, Ostrer H, Harlap S, Stone A, Winters M, Ambrosone CB. Genetic variants in SOD2, MPO, and NQO1, and risk of ovarian cancer. Gynecol Oncol. 2004;93(3):615-20.

63. Mustea A, Heinze G, Sehouli J, Koensgen D, Wolf A, Gutu L, Sofroni D, Pirvulescu C, Braicu El, Schuster E, et al. The -463G/A polymorphism in myeloperoxidase gene and cervical cancer. Anticancer Res. 2007;27(3b):1531-5.

64. Oliveira ID, Petrilli AS, Tavela MH, Zago MA, de Toledo SR. TNF-alpha, TNFbeta, IL-6, IL-10, PECAM-1 and the MPO inflammatory gene polymorphisms in osteosarcoma. J Pediatr Hematol Oncol. 2007;29(5):293-7.

65. Buch SC, Nazar-Stewart V, Weissfeld JL, Romkes M. Case-control study of oral and oropharyngeal cancer in whites and genetic variation in eight metabolic enzymes. Head Neck. 2008;30(9):1139-47.

66. Choi JY, Neuhouser ML, Barnett MJ, Hong CC, Kristal AR, Thornquist MD, King IB, Goodman GE, Ambrosone CB. Iron intake, oxidative stress-related genes (MnSOD and MPO) and prostate cancer risk in CARET cohort. Carcinogenesis. 2008;29(5):964-70.
67. Guo X, Zeng Y, Deng H, Liao J, Zheng Y, Li J, Kessing B, O'Brien SJ. Genetic polymorphisms of CYP2E1, GSTP1, NQO1 and MPO and the risk of nasopharyngeal carcinoma in a Han Chinese population of southern china. BMC Res Notes. 2010;3:212

68. Hsieh YY, Chang CC, Wang YK, Hsu KH, Chen CP, Hsu CM, Tsai FJ. Insulinlike growth factors II exon 9 and E-cadherin-Pml I but not myeloperoxidase promoter-463, urokinase-ApaL I nor xeroderma pigmentosum polymorphisms are associated with higher susceptibility to leiomyoma. Anticancer Res. 2010;30(6):2203-8.

69. Wu SH, Lee KW, Chen CH, Lin CC, Tseng YM, Ma H, Tsai SM, Tsai LY. Epistasis of oxidative stress-related enzyme genes on modulating the risks in oral cavity cancer. Clinica chimica acta; international journal of clinical chemistry. 2010;411(21-22):1705-10.

70. Tefik T, Kucukgergin C, Sanli O, Oktar T, Seckin S, Ozsoy C. Manganese superoxide dismutase lle58Thr, catalase C-262 T and myeloperoxidase G$463 \mathrm{~A}$ gene polymorphisms in patients with prostate cancer: relation to advanced and metastatic disease. BJU Int. 2013;112(4):E406-14.

71. Castillo-Tong DC, Pils D, Heinze G, Braicu I, Sehouli J, Reinthaller A, Schuster E, Wolf A, Watrowski R, Maki RA, et al. Association of myeloperoxidase with ovarian cancer. Tumour biology : the journal of the International Society for Oncodevelopmental Biology and Medicine. 2014;35(1):141-8.

72. Zhou YY, Zhang SM, Cai ZG, Zhang H, Wang L, Xu XP, Wu HB. Myeloperoxidase G463A polymorphism and lung cancer risk in Asians: a pooled analysis. Tumour biology : the journal of the International Society for Oncodevelopmental Biology and Medicine. 2013;34(5):3035-9.

73. Zhou C, Luo Q, Qing Y, Lin X, Zhan Y, Ouyang M. Association between MPO-463G > A polymorphism and risk of lung cancer: a meta-analysis. Tumour biology : the journal of the International Society for Oncodevelopmental Biology and Medicine. 2013;34(6):3449-55.

74. Yang JP, Wang WB, Yang XX, Yang L, Ren L, Zhou FX, Hu L, He W, Li BY, Zhu Y, et al. The MPO-463G > A polymorphism and lung cancer risk: a meta-analysis based on 22 case-control studies. PLoS One. 2013;8(6), e65778.

75. Li J, Fu Y, Zhao B, Xiao Y, Chen R. Myeloperoxidase G463A polymorphism and risk of lung cancer. Tumour biology : the journal of the International Society for Oncodevelopmental Biology and Medicine. 2014;35(1):821-9.

76. Huang C, Ma L, Li D. Association between myeloperoxidase G-463A polymorphism and lung cancer risk. Tumour biology : the journal of the International Society for Oncodevelopmental Biology and Medicine. 2014; 35(1):475-81.

77. Bundred NJ, Dover MS, Aluwihare N, Faragher EB, Morrison JM. Smoking and periductal mastitis. BMJ. 1993;307(6907):772-3.

78. Samoszuk MK, Nguyen V, Gluzman I, Pham JH. Occult deposition of eosinophil peroxidase in a subset of human breast carcinomas. Am J Pathol. 1996;148(3):701-6.

\section{Submit your next manuscript to BioMed Central and we will help you at every step:}

- We accept pre-submission inquiries

- Our selector tool helps you to find the most relevant journal

- We provide round the clock customer support

- Convenient online submission

- Thorough peer review

- Inclusion in PubMed and all major indexing services

- Maximum visibility for your research

Submit your manuscript at www.biomedcentral.com/submit
) Biomed Central 